\title{
Simultaneous CH Planar Laser-Induced Fluorescence and Particle Imaging Velocimetry in Turbulent Flames
}

\author{
Jeffrey M. Donbar and James F. Driscoll \\ Department of Aerospace Engineering, University of Michigan \\ Ann Arbor, MI 48109 \\ Campbell D. Carter \\ Innovative Scientific Solutions, Inc. \\ Dayton, $\mathrm{OH} 45440$
}

\begin{abstract}
The present work addresses the question of whether the chemical reaction zone within a turbulent, high-Reynolds-number jet flame is thin-and can be modeled using strained wrinkled laminar flamelet theory-or is thick-and must be modeled using distributed reaction zone theory. The region near the wrinkled instantaneous stoichiometric contour is identified using CH Planar Laser-Induced Fluorescence (PLIF) imaging and the strain on this interface is measured using simultaneous Particle Imaging Velocimetry (PIV) diagnostics. With a separate set of simultaneous images, the relative position of the $\mathrm{CH}$ and $\mathrm{OH}$ radicals is observed using PLIF. It is found that the $\mathrm{CH}$ reaction zone remains thin and rarely exceeds $1 \mathrm{~mm}$, even near the flame tip in a high-Reynolds-number $(18,600)$ jet flame. The mean thickness of the $\mathrm{CH}$ reaction layer $\left(\delta_{\mathrm{CH}}\right)$ increases from 0.3 to $0.8 \mathrm{~mm}$ in the streamwise direction; this is expected because the scalar dissipation rate is known to decrease with downstream distance and should cause a corresponding increase in $\delta_{\mathrm{CH}}$. However, $\delta_{\mathrm{CH}}$ also increases with jet velocity, which is not predicted by theory. Furthermore, the mean strain rates on the stoichiometric contour increase in the streamwise direction, which is contrary to previous predictions. Thus, strain rate does not, in general, scale with the local dissipation rate in a turbulent flame, and this aspect of the counterflow flow analogy is not valid. It is concluded that unsteady laminar flamelet concepts are consistent with most of the present observations, but a method independent of the
\end{abstract}

local dissipation rate is needed to predict the local strain rate.

\section{Introduction}

Often, the analogy is made between segments of turbulent nonpremixed jet flames and a steady laminar counterflow diffusion flame that is selected to have the same strain rate as the turbulent flame segment; particularly, this analogy is used in "laminar flamelet models" $[1,2]$. The flamelet regime, by definition, exists over regions in which the flame front is thinner than the smallest scale of turbulence, and thus the flame front can be modeled as an ensemble of laminar flamelets. For the flamelet analogy to be realistic, several factors must be known:

1 . if the reaction layers are sufficiently thin (rather than thick, distributed zones);

2. if the reaction-layer properties (e.g. radical concentrations, thickness) scale as predicted by counterflow theory;

3. how frequently the layers extinguish or merge; and

4. if the strain on the turbulent reaction layers is proportional to the dissipation rate as in counterflow flames [2].

If all of these conditions are satisfied, it becomes possible to apply several promising modeling ideas which draw heavily on flamelet theory, including the original flamelet model [2], the Local Integral Moment (LIM) model $[3,4]$, the Large Eddy Simulation (LES) model [5], and Direct Numerical Simulation (DNS) [6]. To date, lack of information on the structure of turbulent 
reaction zones has prevented validation of these models.

Most of what is known regarding reactionzone structure comes from Planar Laser-Induced Fluorescence (PLIF) imaging of $\mathrm{OH}$ in turbulent hydrogen-air flames [7-9]. While these images have been revealing, there is some question about the use of $\mathrm{OH}$ as a flame front marker. Because of the relatively slow removal of $\mathrm{OH}$ by three-body collisions [10], $\mathrm{OH}$ can be convected downstream, making it difficult to determine the primary fuel reaction zone. Consequently, $\mathrm{OH}$ is primarily a marker of the radical recombination zone and does not necessarily identify the flame front. $\mathrm{CH}$ and $\mathrm{C}_{2}$ are better markers of the primary reaction zone, since they are created and removed by two-body reactions during primary fuel decomposition.

Previous attempts at $\mathrm{CH}$ imaging in nonpremixed flames have been hampered by low concentrations (tens of ppm or less as opposed to $10^{3}-10^{4} \mathrm{ppm}$ for $\mathrm{OH}$ ) and interfering fluorescence from Polycyclic Aromatic Hydrocarbons (PAHs), a known soot precursor. Consequently, the few studies involving planar imaging of $\mathrm{CH}$ have been limited to premixed flames [11-15] or regions of nonpremixed flames which contain no precursors, e.g., the base region of lifted flames [16-19]. Stårner et al. [20] and Barlow et al. [21], who obtained $\mathrm{CH}$ images up to 30 jet diameters downstream in a piloted flame near extinction, show the only images, to the authors' knowledge, of $\mathrm{CH}$ in the far field of a nonpremixed flame. Overall, the $\mathrm{CH}$ images shown in the aforementioned studies are similar in nature; they all indicate that the $\mathrm{CH}$ is within a thin, often highly contorted layer located slightly to the rich side of stoichiometric.

While no attempts have been made in jet flames, researchers have used Particle Imaging Velocimetry (PIV) to determine strain and stretch in premixed flames. Driscoll et al. [22] determined aerodynamic strain rate, rate of flame stretch, vorticity, and shear strain rate during a flame-vortex interaction. Similarly, Mueller et al. [23] studied repeatable flamevortex interactions from the wrinkled flamelet to local extinction regimes and generated a time history of flame stretch, strain, and vorticity. Nye et al. [24] measured flame curvature and strain rate along a $\mathrm{V}$-flame interacting with a Kármán vortex street. All of these studies were conducted in laminar flames, while PIV in tur- bulent reacting flows, either premixed or nonpremixed, has been used primarily within the past few years $[25,26]$.

In order to record strain rate, conditioned on the reaction zone, simultaneous PIV and PLIF images are required, the feasibility of which has only recently been demonstrated. Frank et al. [27] used PIV and PLIF imaging of biacetyl (a reactant marker) in an unsteady premixed flame to identify velocity vectors relative to scalar contours of fuel fraction. Rehm and Clemens [28] employed PIV and OH PLIF in turbulent planar nonpremixed $\mathrm{H}_{2} / \mathrm{N}_{2}$-air flames, while Hasselbrink et al. [29] investigated a transverse methane jet flame in cross flow; however, no attempt was made in either case to compute local reaction-zone strain rates due to the breadth of the $\mathrm{OH}$ zones. Prior to the present work, no attempts have been made to perform $\mathrm{CH}$ fluorescence simultaneously with PIV.

\section{Experimental Apparatus}

The axisymmetric burner consisted of a central fuel tube surrounded by a coflowing oxidizer stream. The 5-mm-diameter tube had a lip thickness of $1.47 \mathrm{~mm}$ and was positioned approximately $15 \mathrm{~mm}$ downstream of the exit plane of the $150-\mathrm{mm}$-diameter coflow. For all experiments, the coflow velocity was set to 0.3 $\mathrm{m} / \mathrm{s}$. Flow rates of fuel, diluent, and oxidizer were metered with Tylan mass flow controllers. The burner was mounted on a three-dimensional traversing system so that measurements could be taken at various positions from flame base to tip.

Imaging of $\mathrm{CH}$ in nonpremixed flames is difficult for the reasons alluded to earlier. In order to alleviate the PAH-interference problem, nonsooting permanently blue nonpremixed jet flames, where the fuel is heavily diluted ( $70-80 \%$ by volume) with $\mathrm{N}_{2}$ and the oxidizer is pure $\mathrm{O}_{2}$, were used in this study. Two methane flames were studied extensively, corresponding to jet Reynolds numbers of 9,100 and 18,600 ; pertinent parameters for each flame are listed in Table 1. Aside from improving the feasibility of imaging the $\mathrm{CH}$ layer, additional benefits to using permanently blue flames have been realized as well. The pure oxygen coflow produces flames substantially shorter than methane-air flames (60 vs. 200 jet diameters); the resulting flames are in the momentum dominated flow regime, i.e., buoyancy effects are negligible. 
Becker and Yamazaki [30] have developed a nondimensional parameter, $\xi$, based on the Richardson number ( $\mathrm{Ri}$ ), the ratio of buoyant force to momentum flux, in order to quantify the effects of buoyancy. They suggest that a flame is momentum dominated provided that $\xi$ $\leq 5$; as Table 1 indicates both flames are well below this level. The increase in stoichiometric mixture fraction, $Z_{S t}$, also pulls the flame front radially inward to a point just inside the shear layer; here, the turbulence intensity is much higher than found in typical methane-air flames. Lastly, the stability of the permanently blue flames permits them to remain attached to the tube lip even at exit velocities approaching 70 $\mathrm{m} / \mathrm{s}$.

The experimental details have been described elsewhere [31] and will only be briefly reviewed. A Nd:YAG-pumped dye laser was tuned to the $Q_{1}(7.5)$ transition of the $B^{2} \Sigma^{-}-X^{2} \Pi$ $\left(v^{\prime}=0, v^{\prime \prime}=0\right)$ band $(\lambda=390.30 \mathrm{~nm})$ of $\mathrm{CH}$ and fluorescence from the A-X $(1,1),(0,0)$ and B-X $(0,1)$ bands $(\lambda=420-440 \mathrm{~nm})$ was recorded. This approach provides sufficient separation between pump and detection wavelengths to ensure adequate rejection of scattered incident light, with the added advantage of increased fluorescence yield over schemes where the A-X $(0,0)$ band is pumped and the A-X $(0,1)(\lambda \cong$ $489 \mathrm{~nm}$ ) band is detected. The $230-\mu \mathrm{m}$-thick sheet was passed through the center of the jet, and the resulting images collected using a fast Noct-Nikkor $58-\mathrm{mm} / \mathrm{fl} 1.2$ lens and focused onto a Princeton Instruments (PI) intensified charge coupled device (ICCD) array. In order to improve the weak fluorescence signal, the ICCD pixels were binned $2 \times 2$, resulting in an effective spatial resolution of $122 \mu \mathrm{m} / \mathrm{pixel}$. It was imperative to reject the strong scattering from the PIV particles to ensure the quality of the $\mathrm{CH}$ images; this was accomplished through the use of Schott color glass filters KV-418 and BG-1 [31]. Note that for OH PLIF, a second Nd:YAGpumped dye laser was tuned to the $R_{1}(8.5)$ transition of the $A^{2} \Sigma^{+}-X^{2} \Pi(1,0)$ band $(\lambda=281.34$ $\mathrm{nm})$ and fluorescence from the $\mathrm{A}-\mathrm{X}(1,1)$ and $(0,0)$ bands $(\lambda=306-320 \mathrm{~nm})$ was collected with a UV-Nikkor $105-\mathrm{mm} / \mathrm{f} 4.5$ lens and imaged onto a second PI ICCD array (located on the opposite side of the burner from the $\mathrm{CH}$ ICCD). Temporal separation of the $\mathrm{CH}$ and $\mathrm{OH}$ probe lasers was set to $0.5 \mu \mathrm{sec}$.

A standard two-color scheme was used for the PIV; residual "red" dye laser output (616 $\mathrm{nm}$ ) used in producing the $\mathrm{CH}$ excitation beam was used as the red pulse and the 532-nm output of the second Nd:YAG provided the green pulse. Prior to entering the test section, these two sheets were overlapped with the $\mathrm{CH}$ excitation sheet. Time separation between pulses was monitored with a photodiode and digital oscilloscope and set to 5 and $10 \mu \mathrm{s}$ for the $\operatorname{Re}=$ 18,600 and 9,100 flames, respectively. Particle scattering from each sheet was collected onto a single high-resolution (2036 by 3060 pixels, each $9 \mu \mathrm{m}$ square) Kodak DCS 460 color digital camera; advantages of digital PIV and the crosscorrelation scheme used to process the particle images are discussed in detail by Gogineni et al. [32]. Velocity vectors were resolved using 128pixel-square interrogation regions, corresponding to $1.5-\mathrm{mm}$-square regions of physical space. The interrogation regions were $75 \%$ overlapped such that the velocity vectors are spaced every $0.375 \mathrm{~mm}$ on a regular grid. Overall, the fraction of good vectors was typically $90-95 \%$; bad vectors were removed and replaced by an interpolated value from surrounding good vectors. The $75 \%$ overlap provided good vectors in close proximity to each bad vector and thus reduced the interpolation error. Nominal $0.5-\mu \mathrm{m} \mathrm{Al}_{2} \mathrm{O}_{3}$ particles were introduced in both the coflow and fuel tubes at such a level to ensure sufficient seed density while at the same time minimizing interferences with the CH PLIF images.

\section{$\underline{\text { Results }}$}

\section{Reaction Zone Structure and Thickness}

For the purpose of studying reaction zone structure, CH PLIF was performed without PIV and its accompanying seed particles. Two views were obtained at each location for all four flames studied. The side-view orientation includes the standard vertically oriented laser sheet passing through flame center with the ICCD in position to collect fluorescence at $90^{\circ}$. Alternatively, the $45^{\circ}$ view is obtained by rotating the laser sheet $45^{\circ}$ while also rotating the camera $45^{\circ}$ in the same direction, in order to maintain the $90^{\circ}$-collection geometry; by this means a "cross-sectional" view of the flame surface is obtained (the cross section of a cylindrical flame surface would be an ellipse).

The cross-sectional view provides information on potential out of plane curvature in the 
side view as well as insight on the degree of $\mathrm{CH}$ layer wrinkling. A set of $45^{\circ}$-view images taken at $x / \mathrm{L}_{\mathrm{f}, \mathrm{St}}=0.33$ in a $\mathrm{Re}=18,600$ flame are shown in Fig. 1; the field of view for all images is $19.8 \times 27.6 \mathrm{~mm}$. By this location, the turbulence level has created a condition in which the general elliptical nature of the flame base region has been replaced by a wide variety of shapes and sizes. The distribution is evenly split between small structures with sharp curves and structures with larger radius of curvature. Predominantly the regions are thin, and thicker regions can be a result of the layer becoming aligned with the laser sheet.

The side-view $\mathrm{CH}$ images contain both leftand right-hand flame segments, with a field of view equal to $24 \times 36 \mathrm{~mm}$. A set of side-view images from $x / \mathrm{L}_{\mathrm{f}, \mathrm{St}}=0.67$ is provided in Fig. 2 . By this location, the connected reaction zone of Fig. 1 has become "pinched" or fragmented into several pieces. Usually, the "pinching" creates separate upper and lower burning surfaces with void between. The degree of wrinkling and presence of extremely sharp curves is obvious in each of the images. A potential cause for the "pinching" and fragmenting will be discussed in the CH PLIF/PIV section.

By acquiring several hundred side-view images of the $\mathrm{CH}$ layer only, the layer thickness was quantified. Figure 3 shows the mean $\mathrm{CH}$ layer thickness (FWHM), $\delta_{\mathrm{CH}}$, vs. nondimensional flame height for a variety of jet Reynolds numbers. Thickness was determined by taking a horizontal cut through the image and counting consecutive pixels above a threshold corresponding to half the maximum intensity. Hence, thickness is not necessarily measured in a direction normal to the $\mathrm{CH}$ surface, potentially resulting in an inflated value. Also, the finite resolution of the imaging system might lead to some overestimate of $\delta_{\mathrm{CH}}$, particularly on surfaces with a high degree of wrinkling (which is more pronounced in the $\operatorname{Re}=18,600$ flame). Nonetheless, $\delta_{\mathrm{CH}}<1 \mathrm{~mm}$, even at the tip of the $\operatorname{Re}=18,600$ flame. Furthermore, two other trends are distinctly noticeable: 1) the increase in $\delta_{\mathrm{CH}}$ with axial location for all flames and 2) the increase in $\delta_{\mathrm{CH}}$ due to increased jet velocity. The former can be attributed to the decrease in scalar dissipation from base to tip [33-36]; in conjunction with the conversion from a top-hat to Gaussian profile, local gradients must decrease as well, thereby widening any isocontour of mixture fraction. However, the increase in $\delta_{\mathrm{CH}}$ with jet-exit velocity is not predicted by flamelet theory. One possible explanation is that the small-scale structures at higher Reynolds numbers are thickening the $\mathrm{CH}$ layer, i.e., it is not truly flamelet-like, or layer thickening may be a result of unsteady strain effects. Note that the reported $\mathrm{CH}$ thickness is similar to the value of $0.6 \mathrm{~mm}$ measured in a nonpremixed laminar methane-air flame [37].

\section{CH PLIF/PIV Results}

A sample CH PLIF image with overlaid velocity vectors is shown in Fig. 4 . The image was obtained at $x / \mathrm{L}_{\mathrm{f}, \mathrm{st}}=0.67$ in a $\operatorname{Re}=18,600$ flame. The concomitant large stoichiometric mixture fraction, $Z_{S t}=0.56$, and heat release act to retard the velocity decay such that the mean centerline velocity is $\sim 55 \mathrm{~m} / \mathrm{s}$. In order to visualize the vortical structures, $75 \%$ of the mean centerline velocity has been subtracted from all vectors, and thus vectors in the low-speed coflow side are pointing downward. Several features of velocity-field/CH-layer interactions are noticeable, particularly the large structure deforming the top right and the small vortex distorting the middle of the right-hand side of the flame. The upper left-hand side shows a relatively weak $\mathrm{CH}$ region that is being acted upon by two small vortical structures, one from the jet fluid and the other from the oxidizer. The relative position of the reaction zone and the shear layer is evidenced by the close proximity of downward-pointing vectors to the $\mathrm{CH}$ layer.

Figure 5 is also a CH PLIF/PIV image from $x / \mathrm{L}_{\mathrm{f}, \mathrm{St}}=0.67$ in a $\operatorname{Re}=18,600$ flame; however, it shows a pinched $\mathrm{CH}$ layer and the velocity field associated with that phenomenon. Once again, $75 \%$ of the mean centerline velocity has been subtracted from all of the vectors. It appears as though a pair of large-scale structures has merged in order to split the reaction zone. Evidence of a counter-clockwise-rotating structure (associated with the left-hand side) is visible in the central portion of the jet while portions of a clockwise-rotating vortex can be seen in the lower-right portion of the image. Mungal et al. [38] have observed large-scale structures merging to induce quasi-periodic burn out of the flame tip in jet flames; however, the velocity of their structures is roughly half the speed of the structures measure herein.

With a simultaneous CH PLIF image and planar velocity field, determination of the strain rate on the stoichiometric contour is possible 
(and it will be shown that the $\mathrm{CH}$ layer lies close to the stoichiometric contour). For an axisymmetric configuration, the flame-surface strain rate is given by Mueller et al. [23]:

$\varepsilon_{\mathrm{s}}=\frac{\mathrm{u}_{r}}{r}+\mathrm{n}_{x}^{2} \frac{\partial \mathrm{u}_{r}}{\partial r}+\mathrm{n}_{r}^{2} \frac{\partial \mathrm{u}_{x}}{\partial x}-\mathrm{n}_{r} \mathrm{n}_{x}\left(\frac{\partial \mathrm{u}_{r}}{\partial x}+\frac{\partial \mathrm{u}_{x}}{\partial r}\right)$

Here, $r$ and $x$ are the radial and axial coordinates, respectively, while $u$ is the velocity and $n$ is the flame-front normal. After alignment of the PIV and PLIF fields of view, coordinates of the $\mathrm{CH}$ layer center were extracted from the $\mathrm{CH}$ image at $0.375-\mathrm{mm}$ increments, and each discrete $\mathrm{CH}$ flame-front point was matched with the nearest PIV velocity (from the regular PIV grid). Thus, a maximum spatial displacement error in either the axial or radial direction was half the grid spacing or $0.1875 \mathrm{~mm}$. Straight line segments were used to connect these points, and from that line slope a corresponding perpendicular line slope was determined. Using this slope, a normal vector to the wrinkled contour (pointing toward the oxidizer side) was determined. All of the partial derivatives in Eq. (1) were computed using standard central differences on the regularly spaced velocity grid. This procedure was repeated at each $\mathrm{CH}$ coordinate within an image, typically $75-100$, to determine instantaneous strain rate along the entire flame front.

A sub-region of a simultaneous PIV/CH PLIF image taken at $x / L_{f, S t}=1$ in a $\operatorname{Re}=18,600$ flame is shown in Fig. 6. The strong vortex has pulled the flame tip over, turning it almost $180^{\circ}$ from the original flow direction. Velocity spacing and subtraction are the same as Figs. 4 and 5; hence the structure shown is convecting at roughly 13 $\mathrm{m} / \mathrm{s}$. The effect on the $\mathrm{CH}$ layer is most noticeable at the top, inner surface of the flame and left-hand side of the vortex where the signal level is noticeably weaker than the surrounding layer. It is also interesting to note that the image shown in Fig. 6 is similar to computed images of a single vortex interacting with a vertical flame sheet [39].

The instantaneous strain rate on the $\mathrm{CH}$ layer (along the left-hand side of Fig. 6) was determined using Eq. (1) and is shown in Fig. 7 vs. the tangential flame coordinate (S); also shown is the axial velocity along the $\mathrm{CH}$ contour. In the regions of low $\mathrm{CH}$ PLIF signal, the strain rate is on the order of $15,000 \mathrm{~s}^{-1}$, constituting severe extensive strain, yet the flame does not extinguish (this is in agreement with the counterflow calculations to be discussed and shown in Fig. 10). This high strain rate is a result of the large change in axial velocity, over a small distance, coupled with the dramatic change in flame orientation. Conversely, large compressive strain rates $\left(-5,000 \mathrm{~s}^{-1}\right)$ along the bottom coincide with thicker $\mathrm{CH}$ layers. Some caution should be taken when looking at such high strain rates based on PIV measurements, as a velocity error of $\pm 1 \mathrm{~m} / \mathrm{s}$ (out of $60 \mathrm{~m} / \mathrm{s}$ ) between adjacent regions can result in an error of $\pm 2700 \mathrm{~s}^{-1}$ in strain. Regardless, the measurements indicate regions of large extensive and compressive strain within a turbulent flame without flame extinction. It should also be noted that these are instantaneous snapshots and one cannot determine how long these structures have acted or will act on the flame surface. Some indication of the rapid velocity change associated with the vortex can be noted in Fig. 7, as the axial velocity drops from 50 to $20 \mathrm{~m} / \mathrm{s}$ along the flame surface. Especially notable are the large drops $(5-8 \mathrm{~m} / \mathrm{s})$ in the region of $\mathrm{S}=0.9$ which correspond to regions of high strain.

The statistics of the surface-averaged strain rate are quite revealing. Figure 8 shows the average strain rate on the $\mathrm{CH}$ layer; ten images at each of the four measurement locations were analyzed using Eq. (1), yielding 1000 individual strain measurement points. The $\operatorname{Re}=9,100$ flame shows a relatively constant average strain on the $\mathrm{CH}$ layer of $150 \mathrm{~s}^{-1}$; this is in stark contrast to the $\operatorname{Re}=18,600$ flame which displays an increase in average strain with downstream distance. The disparity between the two cases results because the $\operatorname{Re}=9,100$ flame has a more laminar-like $\mathrm{CH}$ structure while the $\mathrm{CH}$ layers in the $\operatorname{Re}=18,600$ flame are more highly wrinkled and contorted. While the averages are all positive (extensive strain), regions of negative strain and corresponding thicker $\mathrm{CH}$ layers are also observed (see lower left portion of Fig. 6 ). However, the extensive strain tends to dominate, especially downstream.

It was previously noted that the far-field strain rate, according to theory, should scale as $x^{-2}$. This theory, however, assumes the same degree of wrinkling everywhere within the flame, which is not observed. The present findings indicate that the local degree of wrinkling can be a dominant factor in determining local strain rate. Further proof of this is that the final term in Eq. (1) is dominant near the 
flame tip. There, the flame orientation is changing rapidly ( $\mathrm{n}_{x}$ and $\mathrm{n}_{r}$ vary greatly), and in addition the surface is adjacent to the shear layer where $\partial u_{x} / \partial r$ is substantial. Clearly, models should predict the degree of wrinkling to reproduce a realistic strain rate along the reaction zone.

The CH/PIV data allows assessment of the relationship between mixture fraction and velocity. The issue is thoroughly discussed by Kuo [40] and is only briefly reviewed herein. In a laminar jet flame, the $x$-momentum equation and the fuel species conservation, equation written in terms of mixture fraction, have identical solutions, provided diffusivities are equal and exit profiles of velocity and mixture fraction are top-hat in nature. With exact similarity between the processes of mass and momentum transfer, the velocity at the stoichiometric surface, $U_{s}$, can be written as $U_{s}=Z_{S t} \cdot U_{0}$, where $\mathrm{U}_{0}$ is the jet exit velocity. For each of the measurement locations in both the $\operatorname{Re}=9,100$ and $\operatorname{Re}=18,600$ flames, $U_{s}$ (axial velocity only, as radial velocities are low) was computed and plotted in Fig. 9; the 1- $\sigma$ deviations for each point are indicated for each point. Figure 9 indicates that the $\mathrm{CH}$ layer (an isocontour of mixture fraction) stabilizes along an isocontour of axial velocity, approximately 24 and $44 \mathrm{~m} / \mathrm{s}$, for the $\operatorname{Re}=9,100$ and $\operatorname{Re}=18,600$ flames, respectively. Both of these values are slightly higher than expected from the above equation (21 and $37 \mathrm{~m} / \mathrm{s}$ ). Regardless, even in turbulent flames it appears as though the mixture fraction and velocity profiles are similar.

\section{Computations and $\mathrm{CH} / \mathrm{OH}$ Imaging}

For purposes of comparison, a series of computations using steady counterflow flames was conducted. The "fuel" side consisted of a fuel-product mixture at $\mathrm{Z}=0.8$, where $\mathrm{Z}_{\mathrm{St}}=$ 0.56 (same as $\operatorname{Re}=18,600$ flame), and the oxidizer was pure $\mathrm{O}_{2}$ at $300 \mathrm{~K}$ (same as the coflow). Figure 10 shows the computed $\mathrm{CH}$-layer thickness (FWHM) and distance from peak $\mathrm{CH}$ mole fraction to $Z_{S t}$ as a function of both strain rate and the scalar dissipation rate at the stoichiometric contour $\left(\chi_{s}\right)$. As the dissipation rate increases, the computed thickness decreases in agreement with the experimental data in Fig. 3. The disparity between measured and calculated $\delta_{\mathrm{CH}}$ (calculated value is smaller) may be a result of the concerns stated in the section (Reaction Zone Structure and Thickness) on ex- perimental determination of $\delta_{\mathrm{CH}}$. As for the distance between the stoichiometric surface and peak $\mathrm{CH}$ level, the separation at low strain rates is $\sim 1.5 \mathrm{~mm}$; however, as the strain rates increase the profiles narrow, and the separation distance at $2,000 \mathrm{~s}^{-1}$ is less than $0.5 \mathrm{~mm}$. Thus, the strain rates measured along the maximum $\mathrm{CH}$ contour are representative of the strain on the stoichiometric contour.

In order to quantify the differences between $\mathrm{CH}$ and $\mathrm{OH}$ imaging, a set of simultaneous images was acquired. Figure 11 shows the combined $\mathrm{CH} / \mathrm{OH}$ images throughout the entire length of a $\operatorname{Re}=18,600$ permanently blue jet flame. The six uncorrelated images were taken at each of the four measurement locations, $x / \mathrm{L}_{\mathrm{f}, \mathrm{St}}=0,1 / 3$, $2 / 3$ (c \& d), and 1 (e \& f). The center of the images corresponds to $x=10,80,160$, and 200 $\mathrm{mm}$ from the jet exit. In $11 \mathrm{a}$ ), both layers are straight and vertically oriented with an occasional "bump" that appears to result from a structure within the jet fluid. Proceeding to 11 b), these "bumps" have increased in magnitude; the $\mathrm{CH}$ layer remains thin while the $\mathrm{OH}$ layer has thickened and shows some signs of being influenced by outer edge effects. The most dramatic changes occur in going from $11 \mathrm{~b}$ ) to $\mathrm{c}$ ). Here, the $\mathrm{CH}$ layer becomes highly distorted with sharp cusps; furthermore, the two $\mathrm{CH}$ flame surfaces nearly coalesce at the top of the image. A thin band of high $\mathrm{OH}$ signal is adjacent to the $\mathrm{CH}$, but the overall $\mathrm{OH}$-layer width has increased significantly and shows distinct internal structure. In $11 \mathrm{~d}$ ), taken at the same axial location as c), the $\mathrm{CH}$ reaction layers have "pinched" together resulting in separate upper and lower reaction zones with $\mathrm{OH}$ filling the void between them. In turn, this "pinching" phenomenon leads to structures like those appearing in $11 \mathrm{e}$ ); here, small pockets of $\mathrm{OH}$ are surrounded by thin $\mathrm{CH}$ layers within the burning jet cone. It is theorized that these islands are partially premixed pockets that are created during the "pinching" process. Finally, Fig. 11 f) corresponds to the instantaneous stoichiometric flame tip, $\mathrm{L}_{\mathrm{f}}, \mathrm{st}$; as expected, significant quantities of $\mathrm{OH}$ persist downstream in the hot-gas region.

Figure 11 illustrates the discrepancy in thickness between $\mathrm{CH}$ and $\mathrm{OH}$ regions; especially downstream, the $\mathrm{CH}$ layer remains thin and flamelet-like while the $\mathrm{OH}$ layer becomes several $\mathrm{cm}$ thick, more like a distributed reaction zone. In contrast to the findings of several 
studies of lifted hydrocarbon and diluted hydrogen flames [16, 34, 41], no "holes" are seen at the base of the flame; in lifted flames, vortical structures grow within the mixing layer resulting in flame-surface holes [16]. As the flames in this study are attached, and perhaps owing to the increased stability of permanently blue flames [42], this phenomenon is not observed. Furthermore, in the far field there is an abundance of $\mathrm{OH}$ radicals, making it difficult to extinguish the flame front, regardless of strain rate.

\section{Conclusions}

1. The $\mathrm{CH}$ layer remains thin and rarely exceeds $1 \mathrm{~mm}$, even near the flame tip in a high-Reynolds-number $(18,600)$ jet flame. The $\mathrm{CH}$ layer thickness increases in the streamwise direction; this is expected as the scalar dissipation rate has been shown to decrease in the downstream direction of jets. However, $\delta_{\mathrm{CH}}$ also increases with jet velocity, which is not predicted by theory.

2. The $\mathrm{CH}$ layer in turbulent flames is highly contorted and wrinkled, but rarely broken. In the far field, some "pinching" and fragmenting of the $\mathrm{CH}$ layer does occur. It appears as though merging of large-scale structures is responsible for the splintering of the reaction zone further downstream. Clear evidence of the velocity field acting to distort the $\mathrm{CH}$ layer is noted.

3. The strain rates on the stoichiometric contour are small near the flame base and increase in the streamwise direction, contrary to model predictions. The base region does not experience the maximum strain rates, as predicted, because the flame tends to align with the mean flow near the base. Near the tip the flame experiences large extensive strain rates up to $20,000 \mathrm{~s}^{-1}$ where the flame experiences large curvature. The strain on the wrinkled stoichiometric contour does not scale in the same manner as the predicted strain rate on the mean flame contour, and thus it is important for models to simulate the wrinkled flame shape in order to account for effects of strain.

4. On the average, axial velocity along the flame surface is shown to be constant over the entire flame length. This indicates velocity and mixture fraction profiles in turbulent flames are similar as predicted in laminar flames.
5. Simultaneous $\mathrm{CH} / \mathrm{OH}$ imaging clearly demonstrates that these radicals mark different regions within the flame. The $\mathrm{CH}$ layer remains thin and flamelet-like along the entire flame and provides an indication of the primary hydrocarbon consumption zone. On the other hand, $\mathrm{OH}$ is thicker and exhibits definite structure, distributed-like, while providing a better marker of the radical recombination zone.

\section{Acknowledgments}

This work has been supported by the Air Force Research Laboratory, Propulsion Directorate, Wright-Patterson Air Force Base, $\mathrm{OH}$, under AFOSR Contract Nos. F33615-92-C-2202 and F33615-97-C-2702, Dr. Julian Tishkoff, Contract Monitor. JMD has received support from the USAF Palace Knight Program.

\section{References}

1. Peters, N., "Laminar Flamelet Concepts in Turbulent Combustion," Twenty-First Symposium (International) on Combustion, The Combustion Institute, Pittsburgh, PA, 1986, pp. 1231-1250.

2. Peters, N., and Williams, F.A., "Liftoff Characteristics of Turbulent Jet Diffusion Flames," AIAA J. 21:421-429 (1983).

3. Suresh, N.C., Dahm, W.J.A., and Tryggvason, G., "LIM Modeling of Chemical Reactions in Spatially and Temporally Developing Shear Flows", AIAA Paper 940870, Reno, NV, 1994.

4. Tryggvason, G., and Dahm, W.J.A., "An Integral Method for Mixing, Chemical Reactions, and Extinction in Unsteady Strained Diffusion Layers," Combust. Flame 83:207-220, 1991.

5. Grinstein, F.F., and Kailasanath, K., "Three-Dimensional Numerical Simulations of Unsteady Reactive Square Jets," Combust. Flame 100:2-10, 1995.

6. Mahalingam, S., Chen, J.H., and Vervisch, L., "Finite Rate Chemistry and Transient Effects in Direct numerical Simulations of Turbulent Nonpremixed Flames," Combust. Flame 102:285-297,1995.

7. Seitzman, J.M., Üngüt, A., Paul, P.H., and Hanson, R.K., "Imaging and Characterization of $\mathrm{OH}$ Structures in a Turbulent Nonpremixed Flame," Twenty-Third 
Symposium (International) on Combustion, The Combustion Institute, Pittsburgh, PA, 1990, pp. 637-644.

8. Johnson, A.W., Sreenivasan, K.R., and Winter, M., "The Thickness Distribution of $\mathrm{OH}$ Regions in a Turbulent Diffusion Flame," Combust. Sci. Tech. 89:1-7, 1993.

9. Clemens, N.T., Paul, P.H., and Mungal, M.G., "The Structure of $\mathrm{OH}$ Fields in High Reynolds Number Turbulent Diffusion Flames," accepted for publication: Combust. Sci. Tech.

10. Barlow, R.S., Dibble, R.W., Chen, J.-Y., and Lucht, R.P., "Effect of Damköhler Number on Superequilibrium $\mathrm{OH}$ Concentration in Turbulent Nonpremixed Jet Flames," Combust. Flame 82:235-251, 1990.

11. Chen, Y.-C., and Mansour, M.S., "Simultaneous Rayleigh Scattering and Laser-Induced $\mathrm{CH}$ Fluorescence for Reaction Zone Imaging in High-Speed Premixed Hydrocarbon Flames," Appl. Phys. B 64:599605, 1997.

12. Nguyen, Q.-V., and Paul, P.H., "The Time Evolution of a Vortex-Flame Interaction Observed via Planar Imaging of $\mathrm{OH}$ and $\mathrm{CH}$," Twenty-Sixth Symposium (International) on Combustion, The Combustion Institute, Pittsburgh, PA, 1996, pp. 357-364.

13. Paul, P.H., and Dec, J.E., "Imaging of Reaction Zones in Hydrocarbon-Air Flames by Use of Planar Laser-Induced Fluorescence of CH," Opt. Lett. 19:998-1000, 1994.

14. Tsujishita, M., Ipponmatsu, M., and Hirano, A., "Visualization of the $\mathrm{CH}$ Molecule by Exciting $C^{2} \Sigma^{+}(v=1)$ State in Turbulent Flames by Planar Laser-Induced Fluorescence," Jpn. J. Appl. Phys. 32:5554-5569, 1993.

15. Allen, M.G., Howe, R.D., and Hanson, R.K., "Digital Imaging of Reaction Zones in Hydrocarbon-Air Flames Using Planar Laser-Induced Fluorescence of $\mathrm{CH}$ and $\mathrm{C}_{2}$," Opt. Lett. 11:126-128, 1986.

16. Schefer, R.W., Namazian, M., Filtopoulos, E.E.J., and Kelly, J., "Temporal Evolution of Turbulence/Chemistry Interactions in Lifted, Turbulent-Jet Flames," Twenty-Fifth Symposium (International) on Combustion, The Combustion Institute, Pittsburgh, PA, 1994, pp. 1223-1231.
17. Schefer, R.W., Namazian, M., and Kelly, J., "Stabilization of Lifted Turbulent-Jet Flames," Combust. Flame 99:75-86, 1994.

18. Schefer, R.W., Namazian, M., and Kelly, J., " $\mathrm{CH}, \mathrm{OH}$, and $\mathrm{CH}_{4}$ Concentration Measurements in a Lifted Turbulent-Jet Flame," Twenty-Third Symposium (International) on Combustion, The Combustion Institute, Pittsburgh, PA, 1990, pp. 669-676.

19. Namazian, M., Kelly, J.T., and Schefer, R.W., "Near-field Instantaneous Flame and Fuel Concentration Structures," TwentySecond Symposium (International) on Combustion, The Combustion Institute, Pittsburgh, PA, 1988, pp. 627-634.

20. Stårner, S.H., Bilger, R.W., Dibble, R.W., Barlow, R.S., Fourguette, D.C., and Long, M.B., "Joint Planar CH and OH LIF Imaging in Piloted Turbulent Jet Diffusion Flames Near Extinction," Twenty-Fourth Symposium (International) on Combustion, The Combustion Institute, Pittsburgh, PA, 1992, pp. 341-349.

21. Barlow, R.S., Dibble, R.W., Stårner, S.H., Bilger, R.W., Fourguette, D.C., and Long, M.B., "Reaction Zone Structure in Dilute Methane Jet Flames Near Extinction," AIAA Paper 90-0732, Reno, NV, 1990.

22. Driscoll, J.F., Sutkus, D.J., Roberts, W. L., Post, M.E., and Goss, L.P. "The Strain Exerted by a Vortex on a Flame-Determined from Velocity Field Images," Combust. Sci. and Tech. 96:213-229, 1994.

23. Mueller, C.J., Driscoll, J.F., Reuss, D.L., and Drake, M.C., "Effects of Unsteady Stretch on the Strength of a Freely-Propagating Flame Wrinkled by a Vortex," Twenty-Sixth Symposium (International) on Combustion, The Combustion Institute, Pittsburgh, PA, 1996, pp. 347-355.

24. Nye, D.A., Lee, J.G., Lee, T.-W. and Santavicca, D.A., "Flame Stretch Measurements During the Interaction of Premixed Flames and Kármán Vortex Streets Using, PIV," Combust. Flame 105:167-179, 1996.

25. Mungal, M.G., Lourenco, L.M., and Krothapalli, A., "Instantaneous Velocity Measurements in Laminar and Turbulent Premixed Flames Using On-Line PIV," Combust. Sci. Tech. 106:239, 1995.

26. Muñiz, L., and Mungal, M.G., "Instantaneous Flame-Stabilization Veloci- 
ties in Lifted-Jet Diffusion Flames," Combust. Flame 111:16-31, 1997.

27. Frank, J.H., Lyons, K.M., and Long, M. B., "Simultaneous Scalar/Velocity Field Measurements in Turbulent Gas-Phase Flows," Combust. Flame 107:1-12, 1996.

28. Rehm, J.E., and Clemens, N.T., "A PIV/PLIF Investigation of Turbulent Planar Non-Premixed Flames," AIAA Paper 97-0250, Reno, NV, 1997.

29. Hasselbrink, E.F., Mungal, M.G., and Hanson, R.K., "Planar Velocity Measurements and $\mathrm{OH}$ Imaging in a Transverse Jet," AIAA Paper 97-0118, Reno, NV, 1997.

30. Becker, H.A., and Yamazaki, S., "Entrainment, Momentum Flux and Temperature in Vertical Free Turbulent Diffusion Flames," Combust. Flame 33:123$149,1978$.

31. Carter, C.D., Donbar, J.M., and Driscoll, J.F., "Simultaneous $\mathrm{CH}$ Planar LaserInduced Fluorescence and Particle Imaging Velocimetry in Turbulent Nonpremixed Flames," App. Phys. B, 66:129-132 (1998).

32. Gogineni, S., Goss, L., Pestian, D., and Rivir, R., "Two-Color Digital PIV Employing a Single CCD Camera," Accepted for publication: Expts. in Fluids.

33. Nandula, S.P., Brown, T.M., and Pitz, R.W., "Measurements of Scalar Dissipation in the Reaction Zones of Turbulent Nonpremixed $\mathrm{H}_{2}$-Air Flames," Combust. Flame 99:775-783, 1995.

34. Effelsberg, E., and Peters, N., "Scalar Dissipation Rates in Turbulent Jets and Jet Diffusion Flames," Twenty-Second Symposium (International) on Combustion, The Combustion Institute, Pittsburgh, PA, 1988, pp. 693-700.

35. Everest, D.A., Driscoll, J.F., Dahm, W.J.A., and Feikema, D.A., "Images of the Temperature Field and Temperature Gradients to Quantify Mixing Rates within a NonPremixed Turbulent Jet Flame," Combust. Flame 101:59-68, 1995.

36. Feikema, D.A., Everest, D.A., and Driscoll, J.F., "Images of Dissipation Layers to Quantify Mixing Within a Turbulent Jet," AIAA J. 34:2531-2538, 1996.

37. Norton, T.S., and Smyth, K.C., "LaserInduced Fluorescence of $\mathrm{CH}$ in a Laminar $\mathrm{CH}_{4} /$ Air Diffusion Flame: Implications for Diagnostic Measurements and Analysis of
Chemical Rates," Combust. Sci. Tech. 76:120, 1991.

38. Mungal, M.G., Karasso, P.S., and Lozano, A., "The Visible Structure of Turbulent Jet Diffusion Flames: Large-Scale Organization and Flame Tip Oscillation," Combust. Sci. and Tech. 76:165-185, 1991.

39. Ashurst, W.T., and Williams, F.A., "Vortex Modification of Diffusion Flamelets," Twenty-Third Symposium (International) on Combustion, The Combustion Institute, Pittsburgh, PA, 1990, pp. 543-550.

40. Kuo, K.K., Principles of Combustion, John Wiley and Sons, New York, 1986.

41. Chen, T.H., and Goss, L.P., "Statistical $\mathrm{OH}-Z$ one Structures of Turbulent Jet Flames from Liftoff to Near-Blowout," Combust. Sci. Tech. 79:311-324, 1991

42. Du, J., and Axelbaum, R.L., "The Effects of Flame Structure on Extinction of $\mathrm{CH}_{4}$ $\mathrm{O}_{2}-\mathrm{N}_{2}$ Diffusion Flames," Twenty-Sixth Symposium (International) on Combustion, The Combustion Institute, Pittsburgh, PA, 1996, pp. 1137-1142.

TABLE 1. Characteristics of two flames studied in this experiment. $\mathrm{U}_{0}$ is jet exit velocity, $\mathrm{X}_{\mathrm{CH} 4}$ and $X_{\mathrm{N} 2}$ are mole fractions of methane and nitrogen in fuel mixture, $L_{f}$, vis and $L_{f,} S_{t}$ are visible and stoichiometric flame lengths, respectively, $Z_{S t}$ is stoichiometric mixture fraction, and $\xi$ quantifies the effects of buoyancy.

\begin{tabular}{|l|c|c|}
\hline & $\operatorname{Re}=9,100$ & $R e=18,600$ \\
\hline$U_{0}(\mathrm{~m} / \mathrm{s})$ & 31.2 & 65.2 \\
\hline$X_{\mathrm{CH}_{4}}$ & 0.194 & 0.297 \\
\hline $\mathrm{X}_{\mathrm{N} 2}$ & 0.806 & 0.703 \\
\hline $\mathrm{L}_{\mathrm{f} \text { vis }}(\mathrm{mm})$ & 400 & 320 \\
\hline $\mathrm{L}_{\mathrm{f} S t}(\mathrm{~mm})$ & 300 & 240 \\
\hline $\mathrm{Z}_{\mathrm{St}}$ & 0.67 & 0.56 \\
\hline$\xi=\mathrm{Ri}^{1 / 3} \cdot(\mathrm{x} / \mathrm{d})$ & 2.50 & 1.25 \\
\hline
\end{tabular}



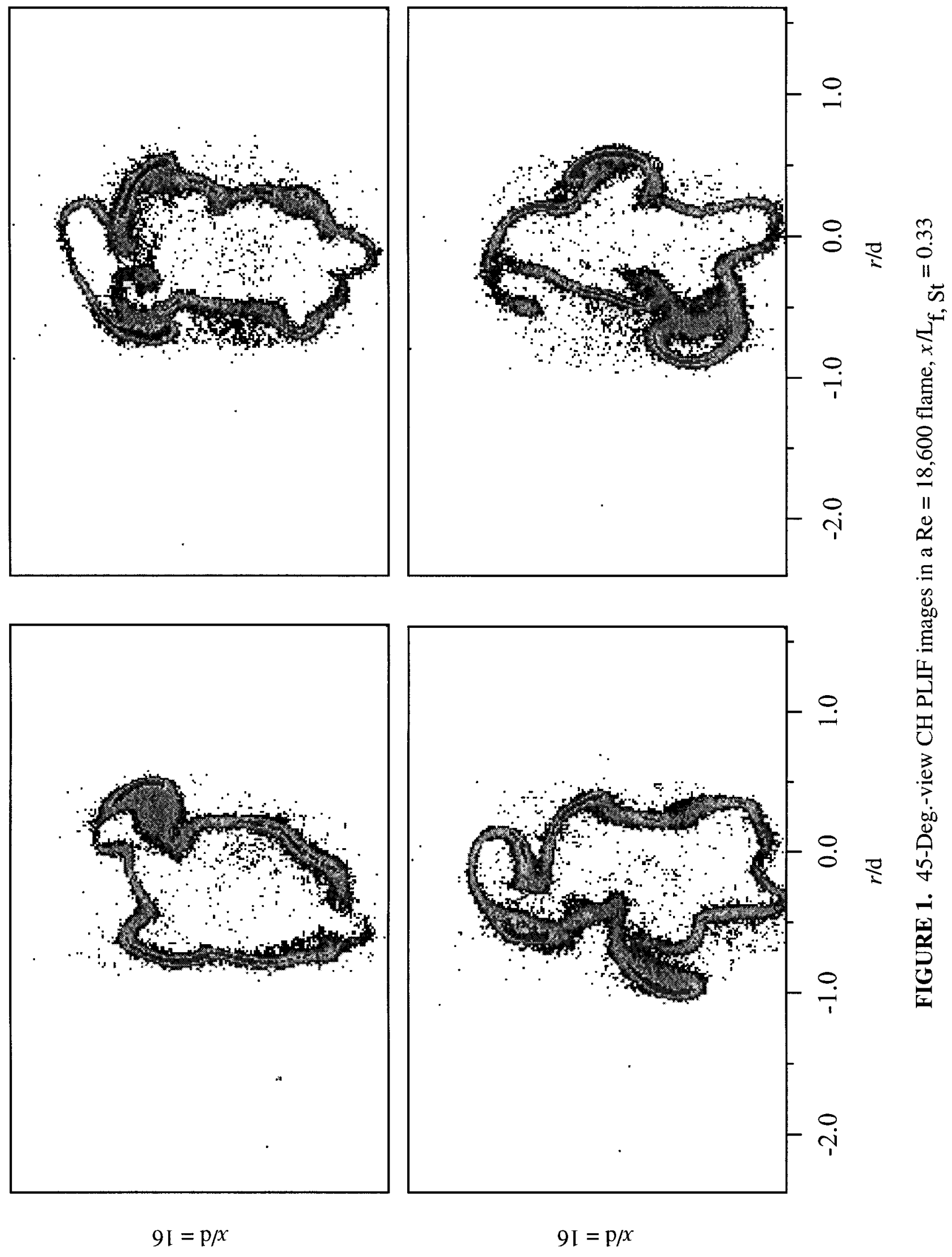

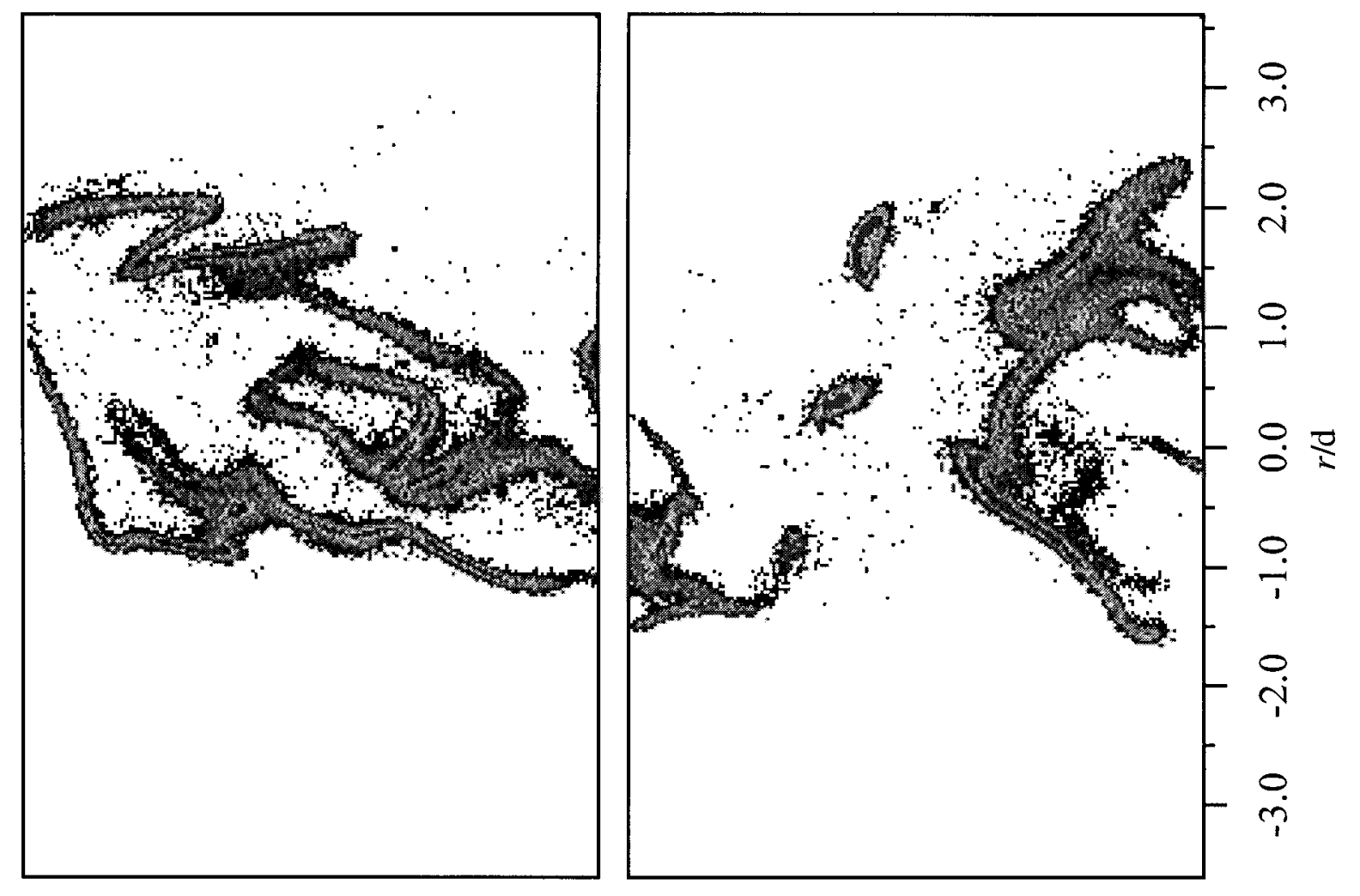

ڤ్ర

ஸे
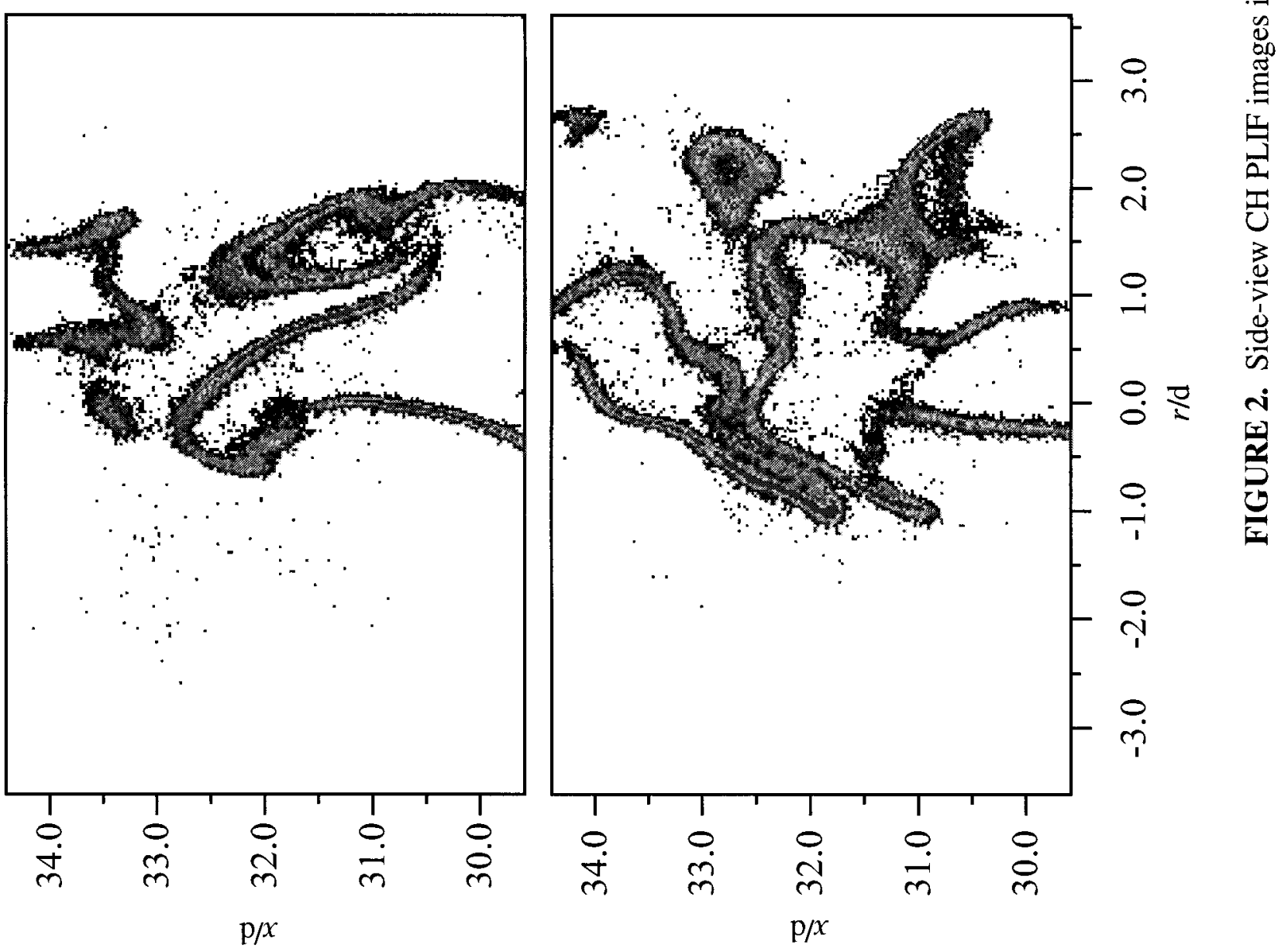


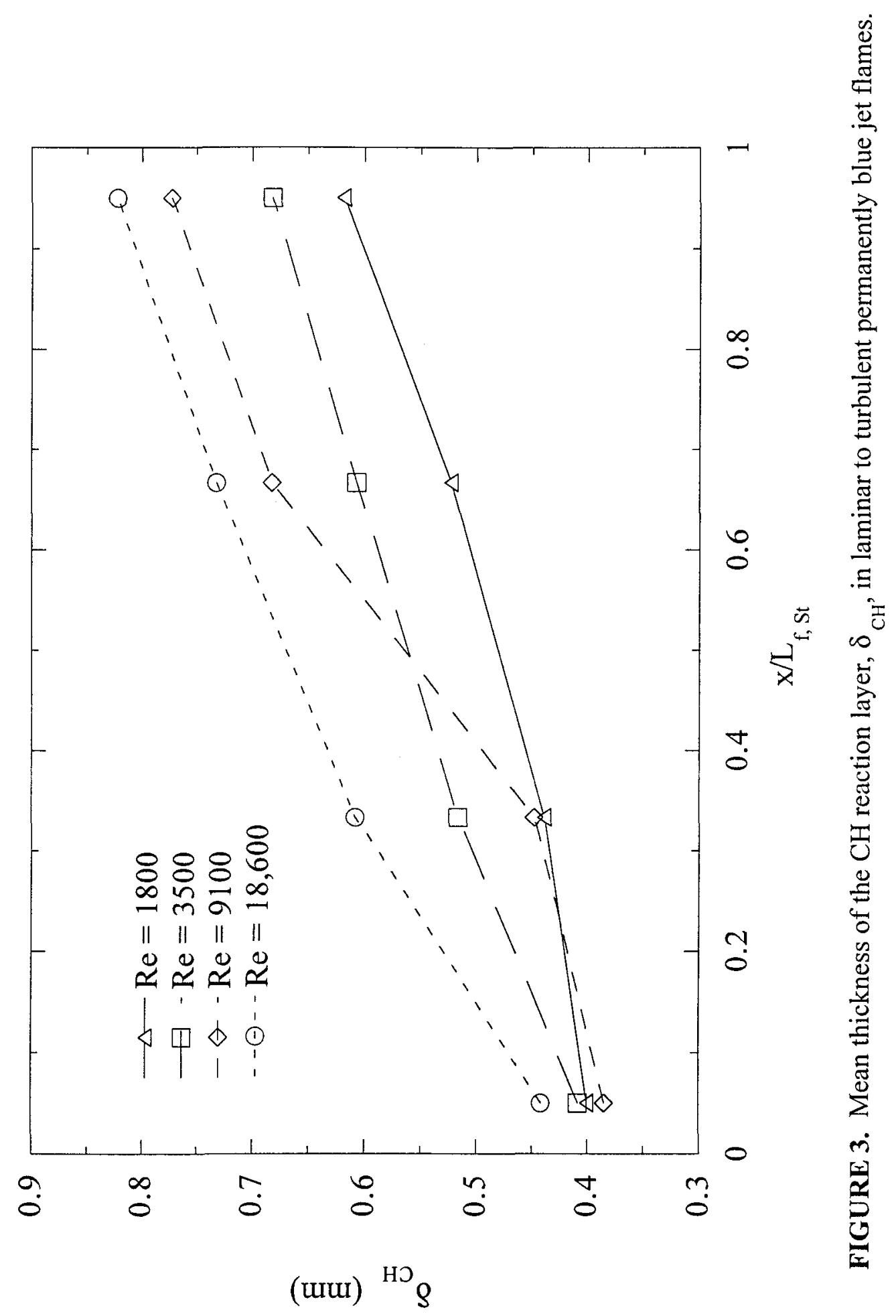




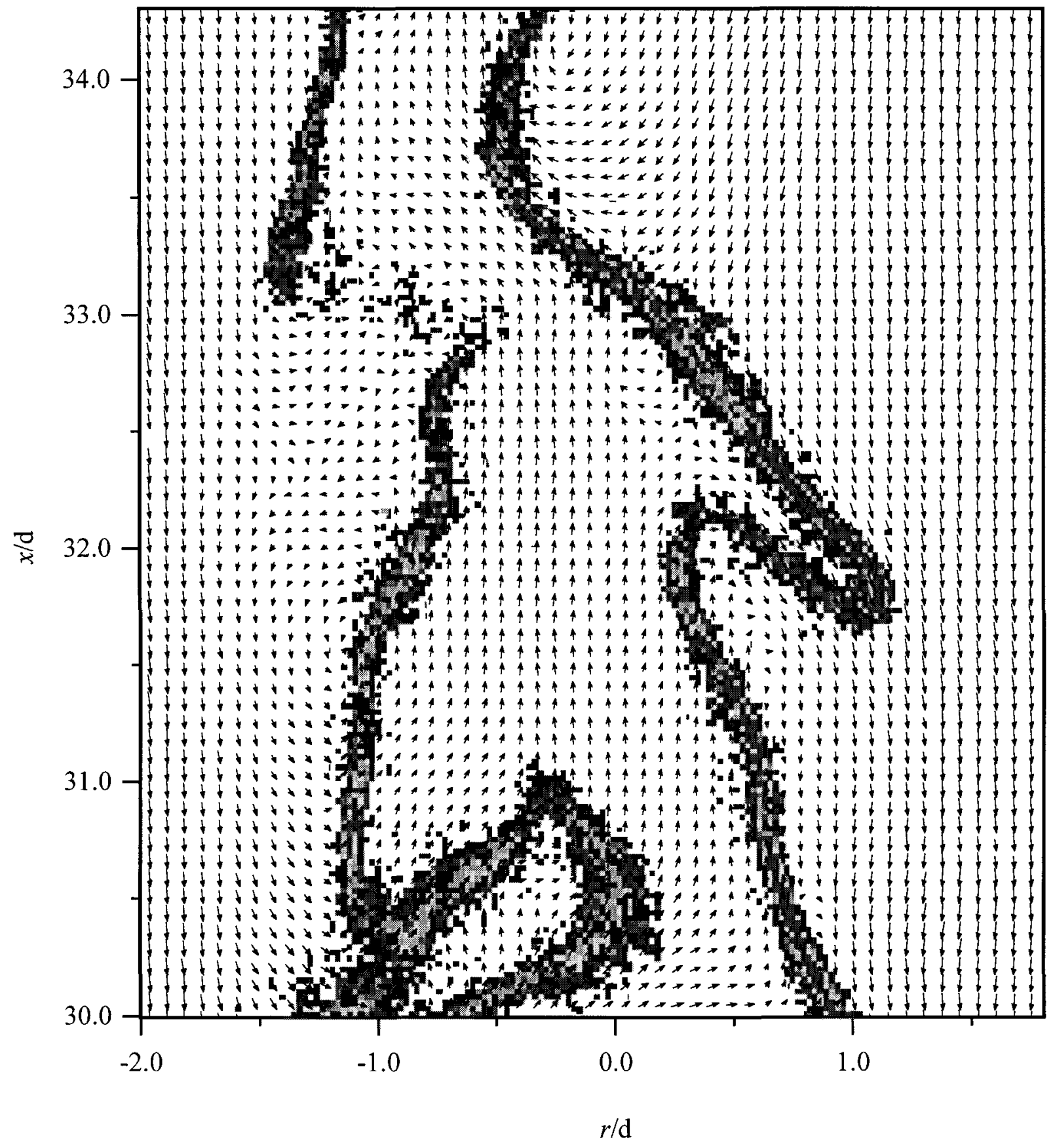

FIGURE 4. Simultaneous CH PLIF/PIV image in a $\mathrm{Re}=18,600$ flame, $x / \mathrm{L}_{\mathrm{f}, \mathrm{St}}=0.67$. Velocity vectors spaced every $375 \mu \mathrm{m}$ on a regular grid and regions are $75 \%$ overlapped; $75 \%$ of the mean centerline velocity has been subtracted from all vectors. 


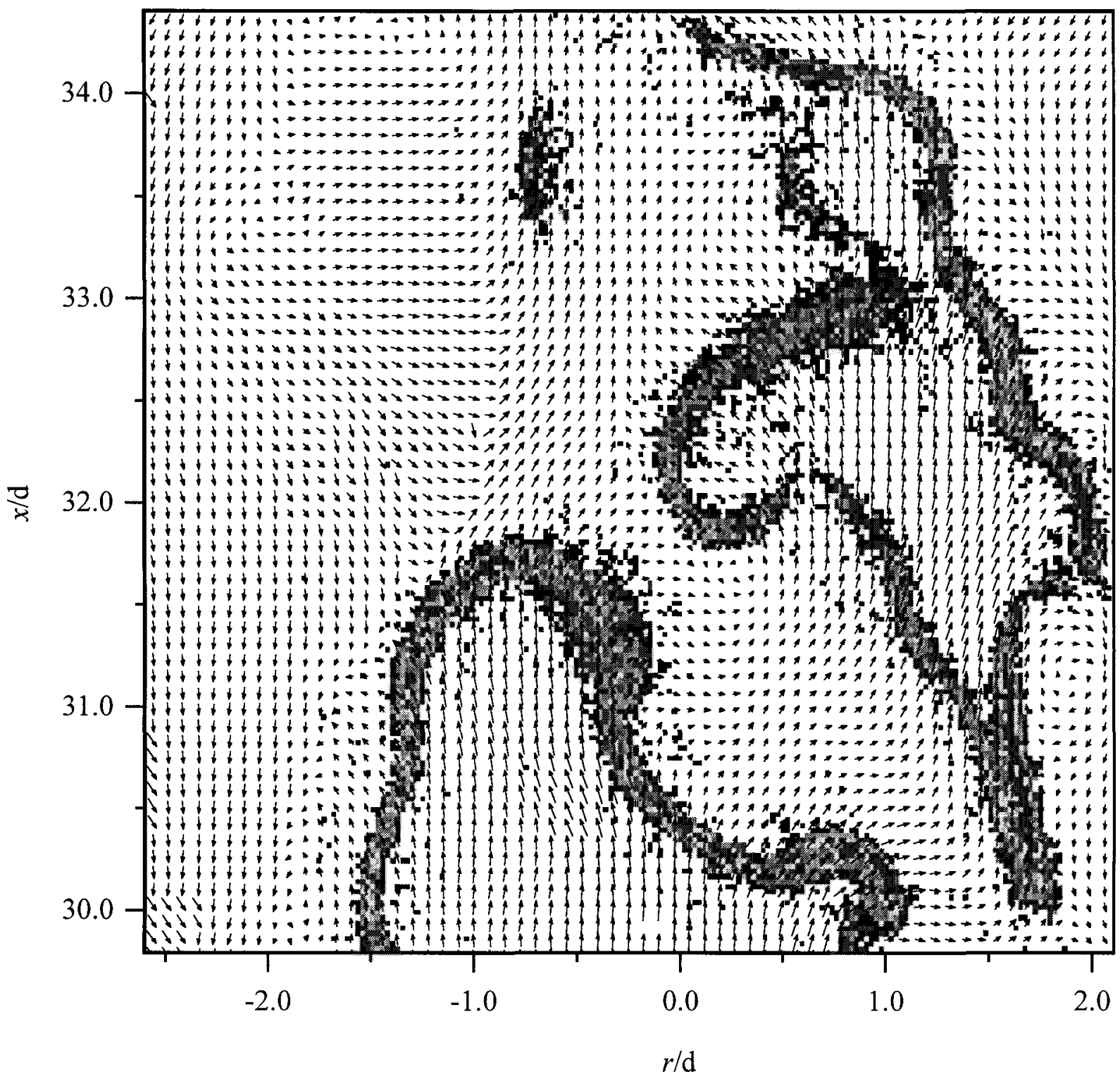

FIGURE 5. Simultaneous CH PLIF/PIV image in a $\mathrm{Re}=18,600$ flame, $x / \mathrm{L}_{\mathrm{f}, \mathrm{St}}=0.67$.

Velocity vectors spaced every $375 \mu \mathrm{m}$ on a regular grid and regions are $75 \%$ overlapped; $75 \%$ of the mean centerline velocity has been subtracted from all vectors. 


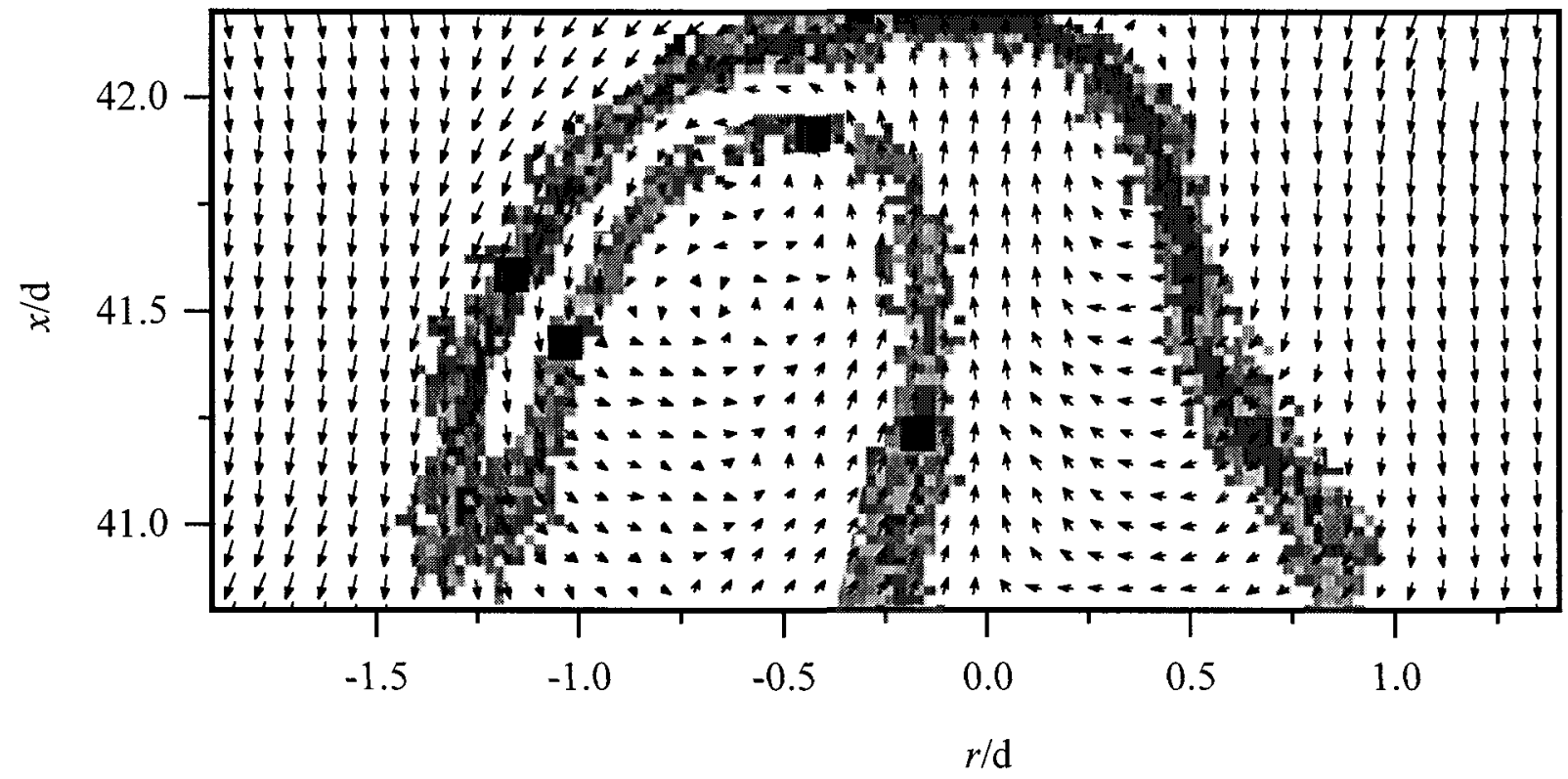

FIGURE 6. Sub-region of a simultaneous $\mathrm{CH}$ PLIF/PIV image in a $\mathrm{Re}=18,600$ flame at $x / \mathrm{L}_{\mathrm{f}, \mathrm{St}}=1.75 \%$ of mean centerline velocity has been subtracted from all vectors.

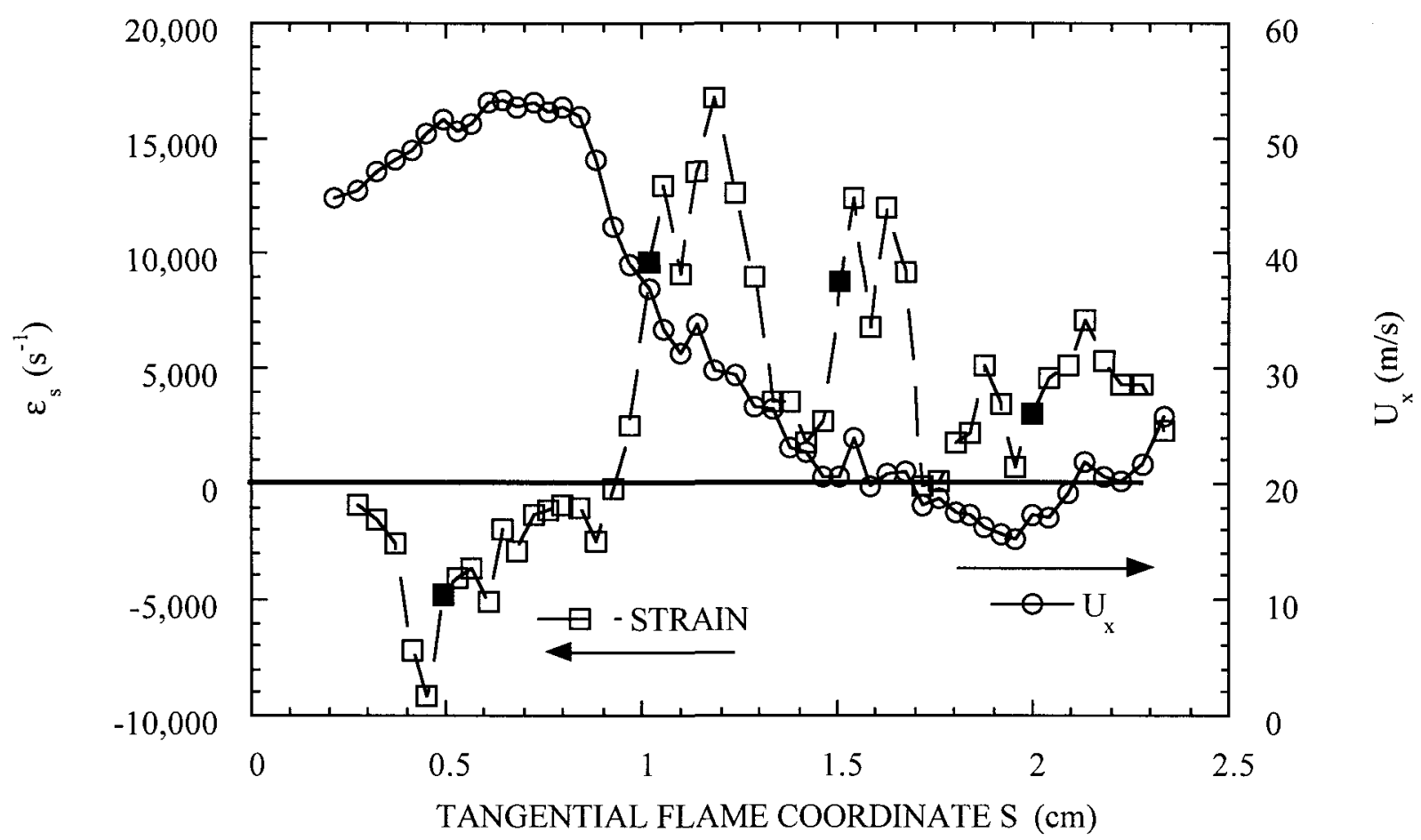

FIGURE 7. Instantaneous flame strain rate, $\varepsilon_{\mathrm{s}}$, and axial velocity vs. flame tangential coordinate--i.e., the distance along the flame front--for the image shown in Fig. 6. For reference, the enclosed squares are indicated on both Figs. 6 and 7. 


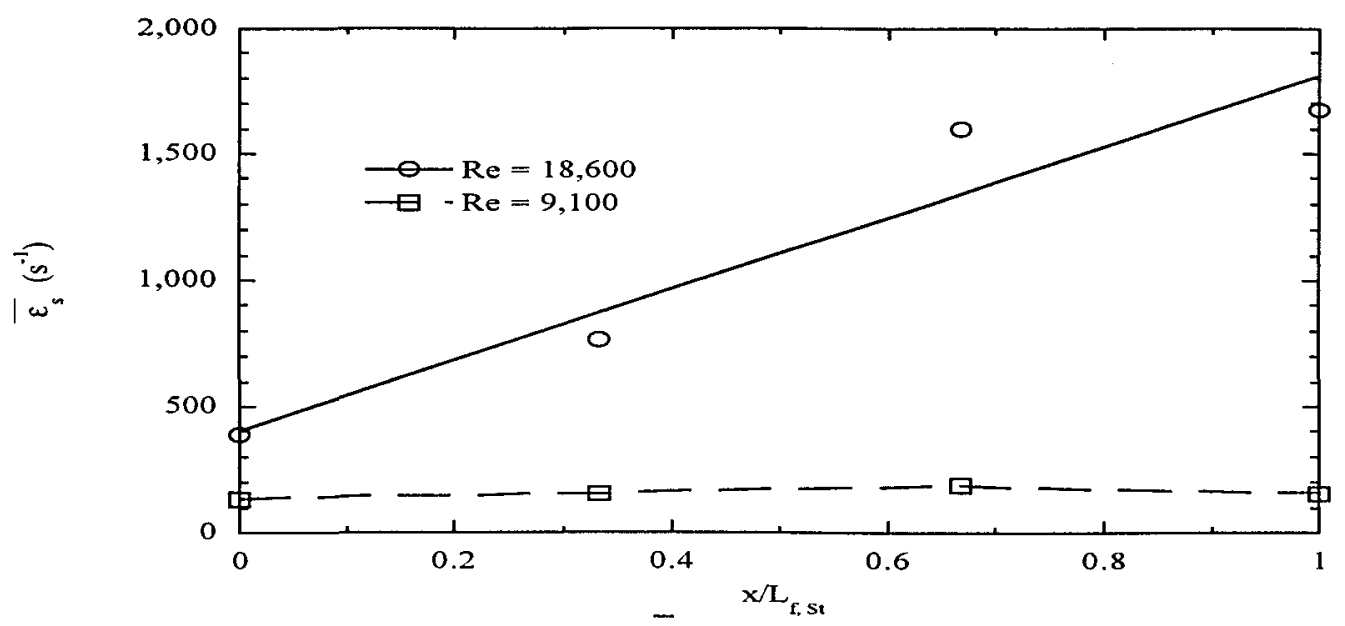

FIGURE 8. Mean strain rate, $\bar{\varepsilon}$, measured along the instantaneous $\mathrm{CH}$ layer.

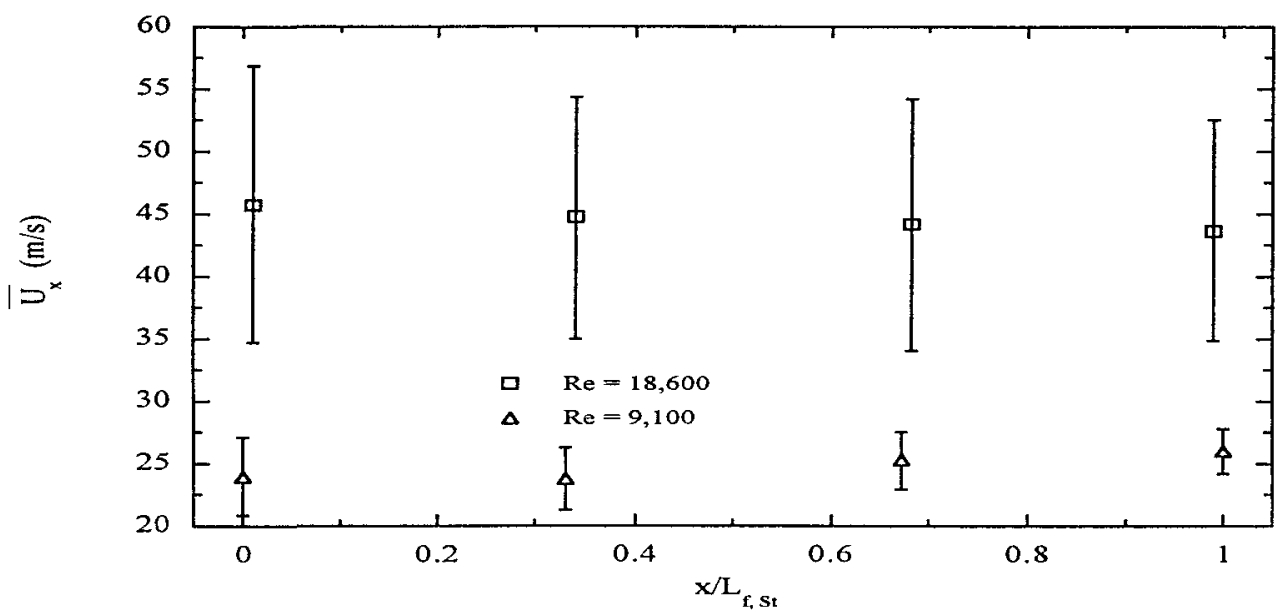

FIGURE 9. Mean axial velocity measured along the instantaneous $\mathrm{CH}$ layer. All points show 1 -s variation in velocity at flame front.

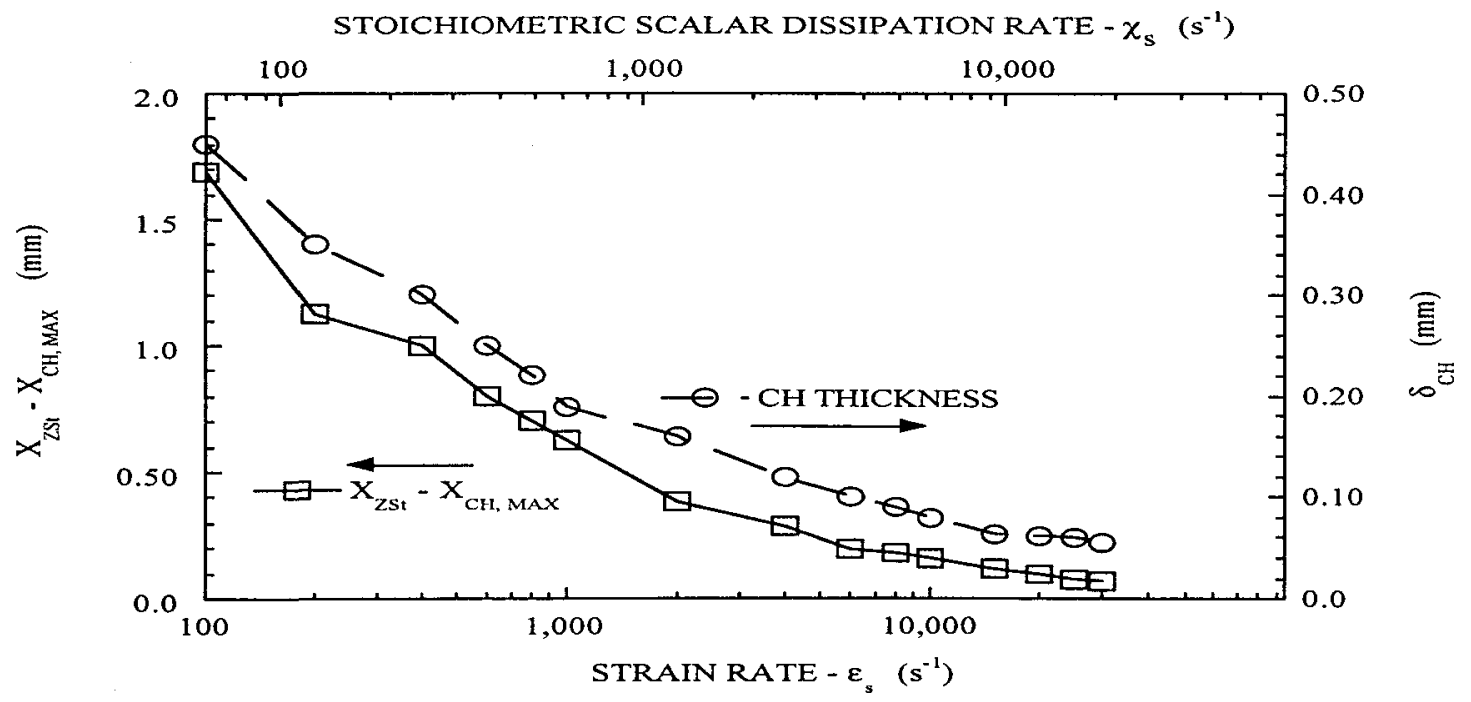

FIGURE 10. Calculated $\mathrm{CH}$ layer thickness and distance from peak $\mathrm{CH}$ mole fraction to stoichiometric contour vs. strain rate, $\varepsilon_{\mathrm{s}}$, and stoichiometric scalar dissipation, $\chi_{\mathrm{s}}$, in steady planar counterflow flames. Fuel-product mixture at $Z=0.8$ and pure oxygen as reactants. 

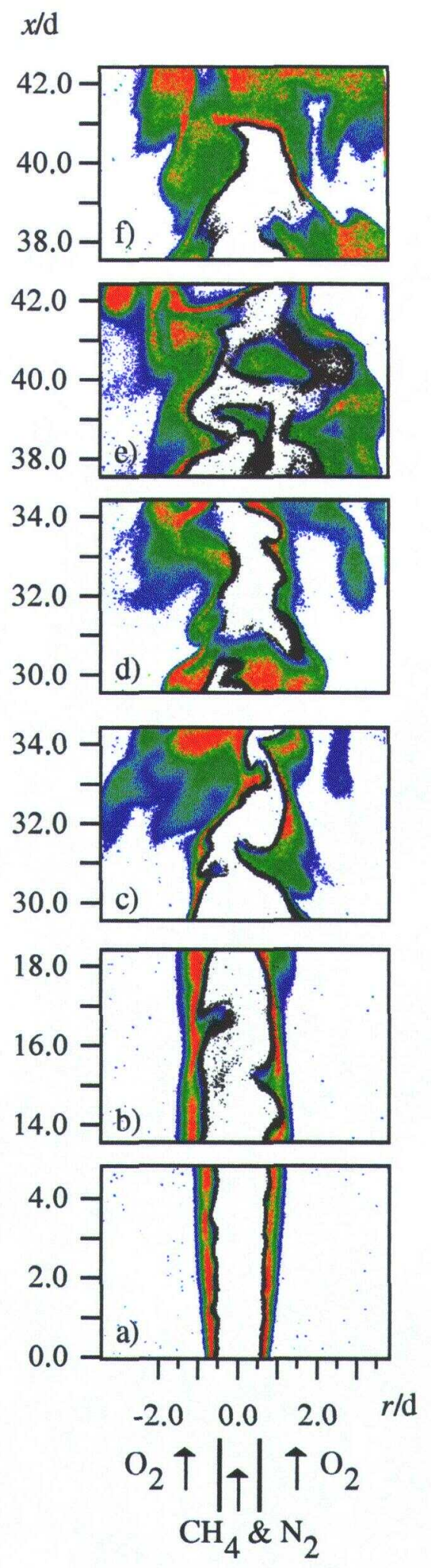

FIGURE 11. Composite image of flame structure for a jet Reynolds number $=18,600$ permanently blue nonpremixed flame. $\mathrm{OH}$ intensity from low (blue) to high (red) with $\mathrm{CH}$ in black (thin layer inside of $\mathrm{OH}$ ). The $x / \mathrm{L}_{\mathrm{f}, \mathrm{St}}$ locations are: a) $0, \mathrm{~b}$ ) $\left.1 / 3, \mathrm{c}\right) 2 / 3$, d) $2 / 3$, e) 1 , and f) 1 . 